\title{
Sleep problems and self-harm in adolescence
}

\author{
Mari Hysing, Børge Sivertsen, Kjell Morten Stormark and Rory C. O'Connor
}

\begin{abstract}
Background
Although self-harm and sleep problems are major public health problems in adolescence, detailed epidemiological assessment is essential to understand the nature of this relationship.
\end{abstract}

\section{Aims}

To conduct a detailed assessment of the relationship between sleep and self-harm in adolescence.

\section{Method}

A large population-based study in Norway surveyed 10220 adolescents aged 16-19years on mental health, including a comprehensive assessment of sleep and self-harm.

\section{Results}

Adolescents with sleep problems were significantly more likely to report self-harm than those without sleep problems. Insomnia, short sleep duration, long sleep onset latency, wake after sleep on set as well as large differences between weekdays versus weekends, yielded higher odds of self-harm consistent with a dose-response relationship. Depressive symptoms accounted for some, but not all, of this association.

\section{Conclusions}

The findings highlight a strong relationship between sleep problems and self-harm. Interventions to reduce adolescent self-harm ought to incorporate sleep problems as a treatment target.

\section{Declaration of interest \\ None.}

\section{Copyright and usage}

(c) The Royal College of Psychiatrists 2015.
Self-harm among adolescents is a major public health concern with approximately $10 \%$ of adolescents having reported self-harm at some stage in their lives. ${ }^{1}$ Although there is growing consensus that we need to look beyond psychiatric disorders to fully appreciate the complexity of the antecedents of self-harm, it remains difficult to predict with acceptable levels of sensitivity and specificity which young people are at elevated risk of selfharm. As a result, there has been a growth in studies focusing on more specific markers of risk. ${ }^{2}$ Such research has identified two main clusters of factors; ${ }^{3}$ environmental influences (e.g. exposure to self-harm) and negative life events on the one hand and psychological factors (e.g. personality and mood) on the other which interact to increase risk of psychological distress and self-harm.

A fruitful strand of the more specific markers of risk research has been the work on self-regulatory processes ${ }^{4}$ including studies on the regulation of sleep. ${ }^{5}$ Attention directed at sleep is unsurprising given the long-standing relationship between sleep and adolescent development in general. ${ }^{6,7}$ However, in recent years there has been growing evidence that sleep problems are risk factors for self-harm and suicidal behaviour and that this relationship is independent of psychiatric disorder. ${ }^{8-12}$ Despite the accumulation of evidence confirming a relationship between sleep problems and self-harm in adolescents, the utility of the findings has been circumscribed because the measures of sleep have tended to be brief. As a consequence, it is not clear whether specific characteristics of sleep disturbance are more strongly associated with risk of self-harm than others. If so, these markers should be highlighted in risk assessment and targeted, if possible, in intervention studies.

The aim of the present study, therefore, was to conduct a detailed investigation of the relationship between sleep problems and self-harm in a large sample of adolescents by employing a more comprehensive assessment of sleep than has been done previously. Given the established relationship between depression, perfectionism and attention-deficit hyperactivity disorder (ADHD) and self-harm, ${ }^{1,13,14}$ we aimed to control for their effects when testing the sleep problem-self-harm relationship.

\section{Method}

In this population-based study, we used data from the youth hordaland-survey of adolescents in the county of Hordaland in Western Norway. All adolescents born between 1993 and 1995 and all students attending secondary education during spring 2012 were invited to participate. The main aim of the survey was to assess the prevalence of mental health problems and service use in adolescents. Data were collected during spring 2012. Adolescents in secondary education received information via email, and time was allocated during regular school hours for completion of the questionnaire. A teacher was present to organise the data collection and to ensure confidentiality. Those not in school received information by postal mail to their home address. Survey staff were available by telephone to answer any queries from both the adolescents and school personnel. The study was approved by the Regional Committee for Medical and Health Research Ethics in Western Norway.

\section{Sample}

A total of 19430 adolescents were invited to participate, of which 10220 agreed, yielding a participation rate of 53\%. Sleep variables were checked for the validity of their answers based on preliminary data analysis, resulting in data from 374 adolescents being excluded due to obvious invalid responses (e.g. negative sleep duration or sleep efficiency). Thus, the total sample size in the current study was 9875 with valid responses on sleep and self-harm variables.

\section{Instruments}

Demographic information

All participants indicated their vocational status, with response options being 'high school student', 'vocational training' or 'not in school'. Maternal and paternal education (highest level) was reported separately with three response options; 'primary school', 
'secondary school' and 'college or university'. Perceived family economy (i.e. how well off they perceive their family to be) was assessed by asking the adolescents how their family economy is compared with most others. Response alternatives were $1=$ 'approximately like most others', 2 = 'better economy' and $3=$ 'poorer economy'.

\section{Self-harm}

Self-harm was assessed using the following question which is taken from the Child and Adolescent Self-harm in Europe (CASE) Study: ${ }^{15}$ 'Have you ever deliberately taken an overdose (e.g. of pills or other medication) or tried to harm yourself in some other way (such as cut yourself)?' If a participant endorsed the 'yes' response, they were asked to complete the following item thinking about the last time they self-harmed if they had self-harmed more than once: 'Describe what you did to yourself on that occasion. Please give as much detail as you can - for example, the name of the drug taken in an overdose'. Classification of self-harm was done according to the CASE guidelines by two coders and in line with the CASE definition of self-harm:

'act with a non-fatal outcome in which an individual deliberately did one or more of the following: initiated behaviour (e.g., self-cutting jumping from a height), which they intended to cause self-harm; ingested a substance in excess of the prescribed or generally recognised therapeutic dose; ingested a recreational or illicit drug that was an act the person regarded as self-harm; ingested a non-ingestible substance or object'

Frequency of self-harm was also recorded and coded as follows: 'none', 'once', 'two or more times'.

A total of 1024 of the 10220 young people completed the open-ended question on self-harm. In $140(1.2 \%)$ patients, the information was not sufficient to code as self-harm, and for 38 $(0.3 \%)$ the description did not meet criteria. This resulted in $846(7.5 \%)$ of the total population meeting the criteria for selfharm. In the current sample, after deleting non-valid responses on sleep, a total of $702(7.2 \%)$ met the criteria for self-harm.

\section{Sleep variables}

Insomnia. Difficulties initiating and maintaining sleep (DIMS) were rated on a 3-point Likert-type scale with response options 'not true', 'somewhat true' and 'certainly true'. If a positive response was endorsed ('somewhat true' or 'certainly true'), participants were then asked how many days per week they experienced problems either initiating or maintaining sleep. Duration of DIMS was rated in weeks (up to 3 weeks), months (up to 12 months) and a last category over a year. A joint question on tiredness/sleepiness was rated on a 3-point Likert-type scale with response options 'not true', 'somewhat true' and 'certainly true'. If there was evidence of tiredness/sleepiness ('somewhat true' or 'certainly true') participants reported the number of days per week they experienced sleepiness and tiredness respectively. Insomnia was operationalised according to the DSM-5 criteria for insomnia: ${ }^{16}$ self-reported DIMS at least three times a week, with a duration of 3 months or more, as well as tiredness or sleepiness at least 3 days per week.

Other sleep variables. Self-reported bedtime and rise time were indicated in hours and minutes using a scroll down menu with 5 -minute intervals and they were reported separately for weekends and weekdays. Time in bed (TIB) was calculated by subtracting bedtime from rise time. Sleep onset latency (SOL) and wake after sleep onset (WASO) were indicated in hours and minutes using a scroll down menu with 5-minute intervals, and sleep duration was defined as TIB - (SOL + WASO). For statistical analyses purposes in the present study, sleep duration was categorised in two different ways (a) 3 groups: 'short sleep' ( $<1$ s.d.: 4.85 h), 'normal sleep' ( $4.85-8 \mathrm{~h}$ ) and 'long sleep' ( $>1$ s.d.: $8 \mathrm{~h}$ ); and (b) 6 groups: ' $<4$ h', ' $4-5$ h', '5-6h', '6-7 h', '7-8 h', ' $8-9$ h', '9-10 h' and ' $10+h$ '. Subjective sleep need was reported in hours and minutes, and sleep deficiency was calculated separately for weekends and weekdays, subtracting total sleep duration from subjective sleep need. Daytime napping was also assessed using the following response alternatives: 'never', 'seldom', 'sometimes', 'mostly' and 'always', with the two latter alternatives coded as positive in a dichotomous variable.

\section{Confounders}

Symptoms of depression were assessed using the short version of the Mood and Feelings Questionnaire (SMFQ). ${ }^{17}$ The SMFQ comprises 13 items assessing depressive symptoms rated on a 3-point Likert-type scale. The wordings of the response categories in the Norwegian translation equates to the original categories of 'not true', 'sometimes true' and 'true'. High internal consistency between the items and a strong uni-dimensionality have been shown in population-based studies, ${ }^{18}$ and have been recently confirmed in a study based on the sample included in the present study. ${ }^{19}$ For the purposes of the current study, depression was defined as a score above the 90th percentile of the Total SMFQ-score. It should be noted that the term depression as used in the current study does not imply existence of a clinical diagnosis, such as major depressive disorder. Also, being a relatively brief self-report questionnaire, the SFMQ does not differentiate between different types of depressive disorders/ conditions. The Cronbach's alpha of the SMFQ in the current study was 0.91 .

Perfectionism was assessed by the short version of the Perfectionism subscale from the Eating Disorder Inventory (EDI). ${ }^{20}$ The scale was adapted to a 3 -point scale from the original 6-point scale for this study with the response options: 'not true', 'somewhat true' and 'certainly true', and a total score was employed for the present study.

Symptoms of ADHD were assessed using the Adult ADHD Self-Report Scale Screener (ASRS). ${ }^{21}$ ASRS is an 18 -item selfreport scale, comprising 9 items on a hyperactivity subscale and 9 items on an inattention subscale reflecting the DSM-IV diagnostic symptom criteria. The response categories are a Likert-type scale ('never', 'rarely', 'sometimes', 'often' and 'very often'). The questionnaire was originally constructed for use with adults, but has recently been validated for use with adolescents. ${ }^{22}$

\section{Statistic}

IBM SPSS Statistics 22 for Mac (SPSS Inc., Chicago, Illinois, USA) was used for all analyses. Pearson's chi-squared test and independent samples $t$-tests were used to examine differences in demographic, psychological and sleep variables between the adolescents reporting self-harm versus no self-harm. Pearson's chi-squared tests were also used to examine differences in sleep problems in method of self-harm (i.e. self-cutting and overdose) and frequency of self-harm (none, once, two or more times). Logistic regression analyses were conducted to examine the predictive effect of the sleep variables (independent variables) on selfharm (dependent variable), using 'no self-harm' as the reference category. Both crude and adjusted models were examined, the latter adjusting for the following covariates entered in separate blocks: (a) demographics (age, gender, parental education and family economy), (b) demographics+depression (SMFQ total score), (c) demographics+perfectionism (EDI total score), (d) demographics+ADHD (ASRS Inattention and ASRS Hyperactivity/Impulsivity subscales, and (e) fully adjusted model (i.e. including all covariates). 


\section{Results}

\section{Demographic characteristics of the sample}

The mean age was 17.9 (s.d. $=0.8$ ) years, and the sample included more girls $(53.3 \%)$ than boys (46.7\%). The majority (98\%) were high-school students. A total of $5.3 \%$ of the sample was defined as immigrants as they had both parents born outside Norway. In terms of maternal education, $10.1 \%$ had been educated to primary school level, $41.3 \%$ to secondary school level and $48.6 \%$ to university/college level. The corresponding percentages for paternal education were $10.6 \%, 46.4 \%$ and $43.0 \%$ respectively. A total of $67.4 \%$ reported having a family economy (income) 'like most others', whereas $25.5 \%$ had 'better' and $7.1 \%$ had 'worse family economy'.

\section{Self-harm}

As detailed in Table 1, 7.2\% of the population met the criteria for self-harm, of which 55\% reported self-harm on two times or more occasions. There were significantly more girls $(11 \%)$ than boys
(2.8\%; $P<0.001)$ meeting the criteria. The most frequent method used was self-cutting $(n=547)$ followed by overdose $(n=115)$ and other methods $(n=17)$. Self-harm was significantly associated with lower parental education, poor family economy, as well as more symptoms of depression, perfectionism and ADHD (all $P<0.001$ ).

\section{Self-harm and sleep}

As depicted in Fig. 1 and detailed in Table 1, there was a significant relationship between self-harm and sleep duration. The average sleep duration among adolescents reporting self-harm was $05.33 \mathrm{~h}$, compared with $06.29 \mathrm{~h}$ among adolescents without no self-harm. Figure 1 shows that a significantly larger proportion of adolescents reporting self-harm slept less than $5 \mathrm{~h}$ compared with no adolescents without self-harm.

Adolescents reporting self-harm also reported significantly longer SOL, WASO and had larger sleep deficiency than their non-self-harming peers (all $P<0.001$ ). The differences between weekdays and weekend bedtimes were also larger among those adolescents who self-harmed $(P=0.023)$ and they also napped more frequently during the day $(P<0.001$; see Table 1 for details).

\begin{tabular}{|c|c|c|c|}
\hline & No self-harm ( $n=9139,92.8 \%)$ & Self-harm $(n=707,7.2 \%)$ & $P$ \\
\hline \multicolumn{4}{|l|}{ Demographic variables } \\
\hline Age, years: mean (s.d.) & $17.9(0.8)$ & $17.8(0.8)$ & 0.004 \\
\hline Gender & & & $<0.001$ \\
\hline Girls, \% (n) & $89.0(4673)$ & $11.0(576)$ & \\
\hline Boys, \% (n) & $97.2(4466)$ & $2.8(128)$ & \\
\hline \multicolumn{4}{|l|}{ Sleep variables } \\
\hline Sleep duration category, \% (n) & & & $<0.001$ \\
\hline Short sleeper (<1 s.d.: 4.85 h) & $13.2(1147)$ & $33.2(232)$ & \\
\hline Normal sleeper & $76.3(6650)$ & $60.7(424)$ & \\
\hline Long sleeper (1>s.d.: 8h) & $10.6(922)$ & $6.0(42)$ & \\
\hline Sleep duration, h:min: mean (s.d.) & $6: 29(1: 36)$ & $5: 33(1: 56)$ & $<0.001$ \\
\hline Insomnia (DSM-V), \% (n) & $16.5(1456)$ & $43.5(307)$ & $<0.001$ \\
\hline Sleep onset latency (SOL), h:min: mean (s.d.) & $0: 45(0: 56)$ & $1: 13(1: 05)$ & $<0.001$ \\
\hline $\mathrm{SOL}>30 \mathrm{~min}, \%(n)$ & 36.9 (3374) & $61.0(431)$ & $<0.001$ \\
\hline Wake after sleep onset (WASO), h:min: mean (s.d.) & 0:13 (0:38) & $0: 33(0: 54)$ & $<0.001$ \\
\hline WASO > $30 \mathrm{~min}, \%(n)$ & $10.8(984)$ & $27.3(193)$ & $<0.001$ \\
\hline Sleep deficiency (>2h), \% (n) & $45.2(3247)$ & $68.2(375)$ & $<0.001$ \\
\hline Bedtime diff (weekdays/weekends) > 2h, \% (n) & $48.7(4276)$ & $53.2(374)$ & 0.023 \\
\hline Daytime napping, \% (n) & $22.0(2008)$ & $35.8(454)$ & $<0.001$ \\
\hline
\end{tabular}

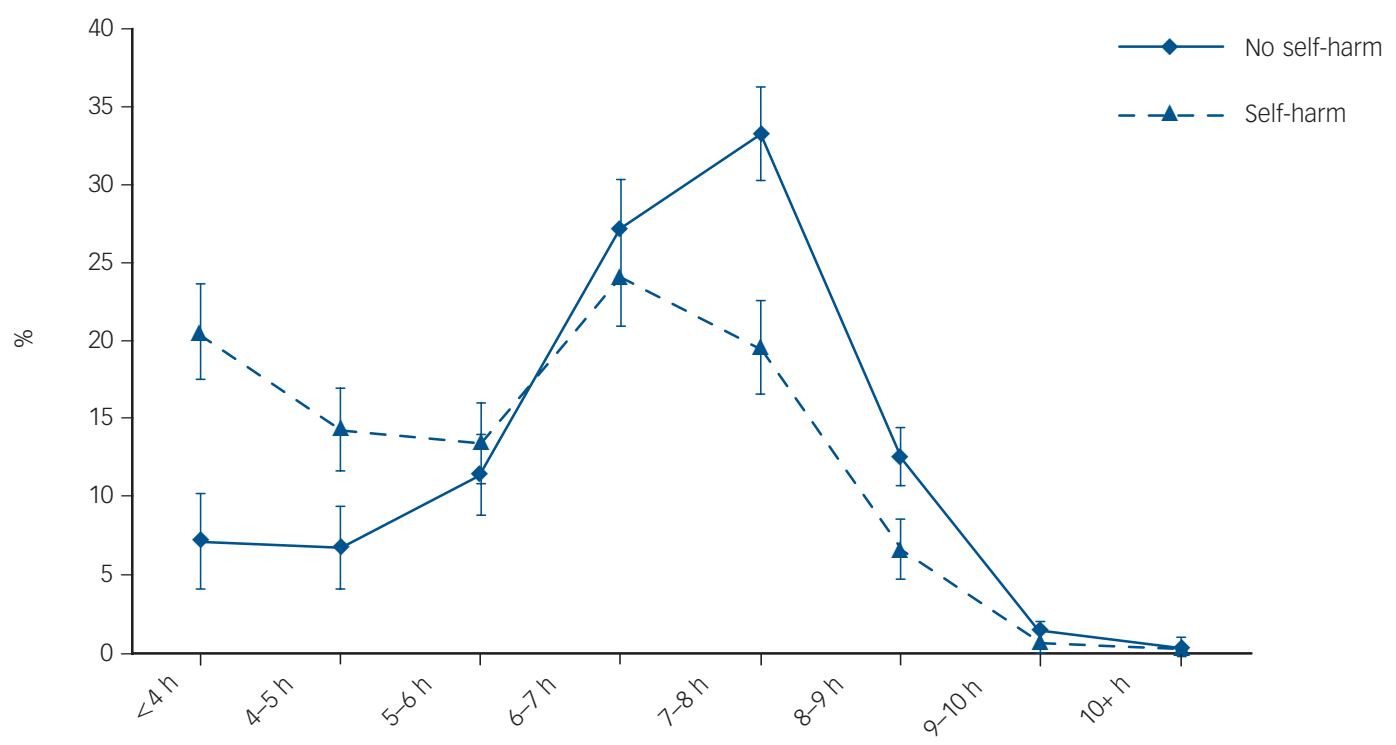

Fig. 1 Self-harm and no self-harm among adolescents stratified by categories of sleep duration. 


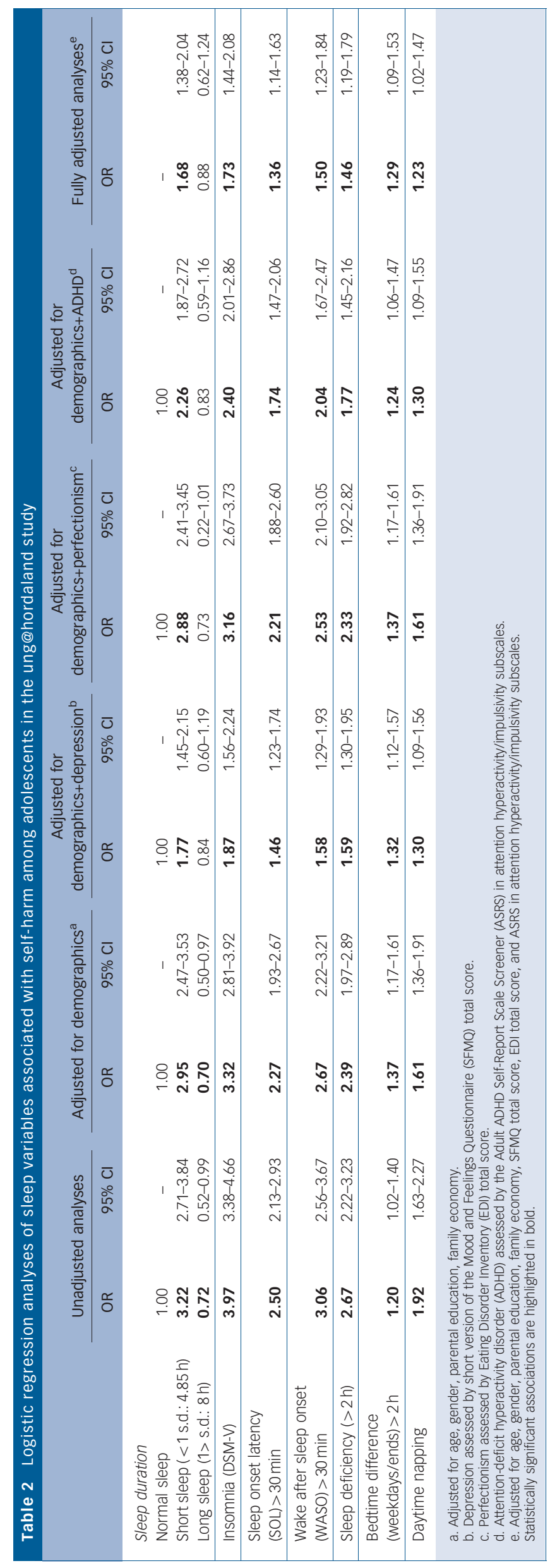

The results from the series of logistic regression analyses investigating the relationship between the different sleep variables and self-harm are presented in Table 2. Reinforcing the findings from Table 1, the crude analyses showed that all sleep variables, except long sleep duration, were associated with significantly increased odds of also reporting self-harm.

As detailed in Table 2, adjusting for potential confounders, including sociodemographics, symptoms of depression, perfectionism and ADHD symptoms, reduced several of the odds ratios (ORs). Depression was the confounder that explained most of the reductions in ORs; sociodemographic factors, perfectionism and ADHD did not, or only slightly, attenuated the associations. However, the effect on all sleep variables remained significant in the fully adjusted analyses: short sleep duration $(\mathrm{OR}=1.68,95 \%$ CI 1.38-2.04), insomnia ( $\mathrm{OR}=1.73,95 \%$ CI $1.44-2.08)$, SOL $(\mathrm{OR}=1.36,95 \%$ CI $1.14-1.63)$, WASO $(\mathrm{OR}=1.50,95 \% \quad \mathrm{CI}$ $1.23-1.84$ ), sleep deficiency ( $\mathrm{OR}=1.46,95 \%$ CI 1.17-1.79), bedtime differences between weekdays and weekends $(\mathrm{OR}=1.29$, 95\% CI 1.09-1.53) and daytime napping (OR $=1.23,95 \%$ CI $1.02-1.47)$.

\section{Method of self-harm and sleep}

The prevalence of insomnia among adolescents reporting an overdose $(n=115)$ was $47 \%$, compared with $43.3 \%$ among those reporting self-cutting $(n=547)$, and $16.6 \%$ of those reporting no self-harm $\left(\chi^{2}(2)=306.6, P<0.001\right)$. Similarly, as depicted in Fig. 2, nearly half of those reporting overdose tend to sleep less than 4.85 h (1 s.d.), compared with $30.6 \%$ among those reporting self-cutting and $13.3 \%$ of those reporting no self-harm $\left(\chi^{2}(4)=206.4, P<0.001\right)$.

\section{Frequency of self-harm and sleep}

There were significant dose-response associations between sleep problems and the frequency of self-harm. As depicted in Fig. 3, the prevalence of insomnia was $48 \%$ among adolescents reporting self-harm two times or more, compared with $37 \%$ among adolescents reporting having engaged in self-harm once $\left(\chi^{2}(2)=423.2, P<0.001\right)$. The same pattern was found for short sleep duration $\left(37 \%\right.$ v. $\left.29 \%\left[\chi^{2}(4)=302.2, P<0.001\right]\right)$, SOL $\left(64 \%\right.$ v. $\left.59 \%\left[\chi^{2}(2)=212.9, P<0.001\right]\right)$ and WASO $(30 \%$ v. $24 \%$ $\left.\left[\chi^{2}(4)=223.9, P<0.001\right]\right)$.

\section{Discussion}

This population-based study of adolescents demonstrated a consistent association between sleep and self-harm, presenting as a dose-response relationship; the more sleep problems, the higher frequency of self-harm. Self-harm was linked to a range of different sleep parameters, including insomnia, short sleep duration, long SOL and WASO, as well as large discrepancies between weekdays versus weekend bedtimes. Whereas depression did account for some of the association between sleep and selfharm, neither perfectionism nor symptoms of ADHD had any impact on the sleep-self-harm association.

Previous studies have heightened our awareness of the importance of sleep in relation to self-harm and suicidal behaviour, but the measures assessing sleep have typically been brief and non-specific, often using a single item to assess symptom of insomnia. The current study extends this literature by assessing a wide range of different sleep problems, including a full operationalisation of the recent DSM-5 criteria of insomnia. Further, no studies have previously examined the link between sleep duration, in contrast to subjectively reported insomnia. Whereas sleep duration and insomnia may indeed overlap, they 


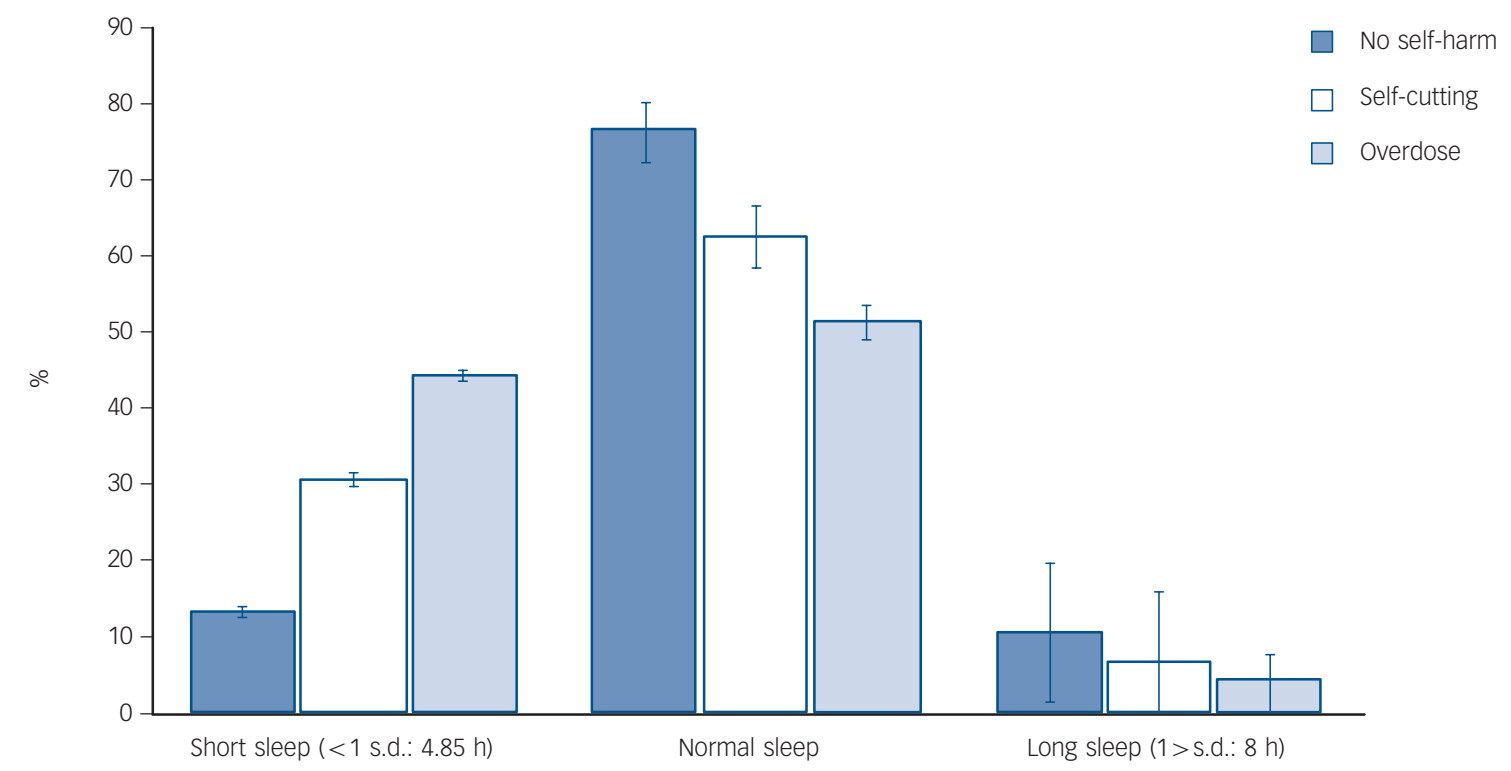

Fig. 2 Sleep duration stratified by type of self-harm.

Error bars represent 95\% confidence intervals. If confidence intervals do not overlap then the difference between the estimates is statistically significant at $P<0.001$.

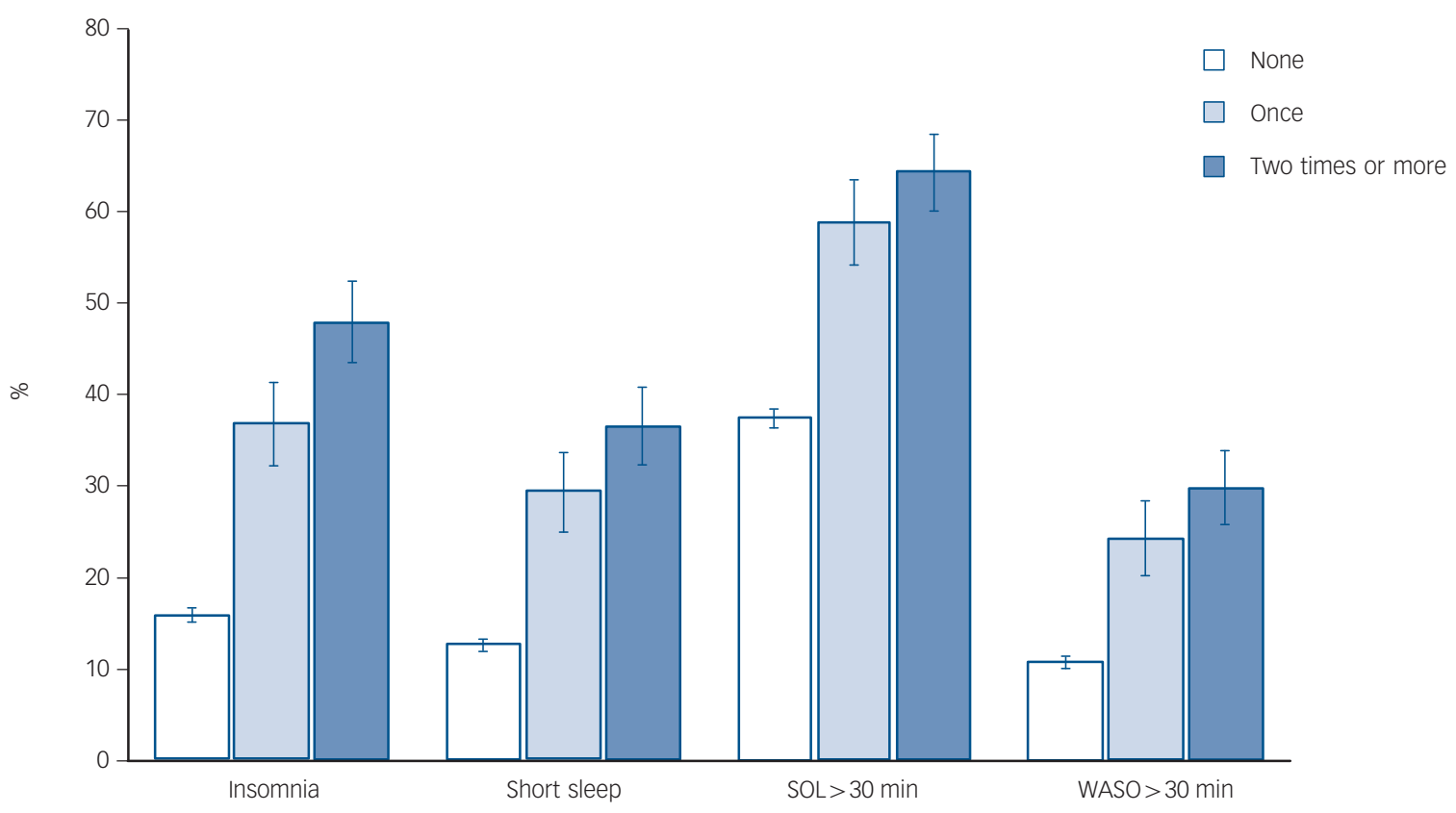

Fig. 3 Self-harm and sleep problems, stratified by frequency of self-harm.

Error bars represent $95 \%$ confidence intervals. If confidence intervals do not overlap then the difference between the estimates is statistically significant at $P<0.001$.

are distinct sleep parameters, with differences in relation to both risk factors and consequences such as school performance and gender patterns. ${ }^{23}$ It is therefore important to assess a wide range of sleep parameters, as conclusions regarding one of them (e.g. insomnia) cannot necessarily be generalised to the others (sleep deficit). However, the results from the current study show that the sleep-self-harm relationship is consistent across a wider range of sleep measures. However, inspection of the overlap in confidence intervals suggests that insomnia is a stronger risk factor than some of the other sleep parameters, such as sleep deficiency, SOL and weekday-weekend differences.

As we expected, adolescents who reported self-harm also had higher rates of depression, perfectionism as well as ADHD symptoms than those who did not report self-harm. Knowing from past research that these factors have been linked to both initiation and maintenance of sleep problems, ${ }^{24-26}$ they may be important factors in accounting for the overlap between self-harm and sleep. As hypothesised, depression accounted for some of the association between sleep and self-harm, but the latter association remained significant even in the fully adjusted analyses, which is consistent with previous studies. ${ }^{5}$ Theoretically, emotional regulation can serve as a useful framework for understanding this relationship. Indeed, it has been suggested that the effect of sleep problems on emotion regulation may be more marked in adolescents than in adults. This was exemplified in an experimental study comparing sleep deprivation on a catastrophising task in three age groups, with adolescents showing a higher rate of catastrophising when sleep deprived than adults. ${ }^{27}$ Symptoms of 
ADHD have also been shown to be related to self-harm ${ }^{28}$ and it has been previously suggested that they may explain the link between sleep and self-harm. ${ }^{5}$ However, this was not supported in the present study, with impulsivity, inattention and hyperactivity not reducing the strength of the association between sleep and self-harm.

The present study indicates that sleep problems and short sleep duration are sensitive markers for self-harm. The findings also suggest that the relationship between sleep and self-harm varies as a function of method of self-harm. In future research, it would be important to determine whether this relationship is moderated by frequency and medical seriousness of self-harm. The general effectiveness of sleep interventions in reducing symptoms of both sleep and co-occurring symptoms suggest that such interventions may play a role in the prevention and treatment of self-harm. Incorporating sleep interventions transdiagnostically to improve self-regulation has been suggested, and has shown promise in reducing symptoms of depression as well as addressing sleep problems. ${ }^{29}$ Recently, including healthy sleep in the prevention of self-harm and suicide has been suggested as one of five factors recommended for future interventions. ${ }^{30}$ However, further studies are required to determine whether including sleep interventions, as part of self-harm treatment or prevention programmes, is effective.

\section{Strengths and limitations}

Although the study has many strengths, there are some potential limitations worth discussing. A limitation of the present study, consistent with other studies which rely on self-report, is that the findings are subject to response biases and may have been affected by demand characteristics. Nonetheless, as far as possible, we have employed widely used questionnaire measures with recognised reliability and validity. The present study is based on a broad and detailed assessment of sleep. Although the definition of insomnia was based on published quantitative criteria, it was not based on a structured interview, which, of course, is difficult to employ in a population-based study. However, the use of both SOL and WASO to estimate exact sleep duration was a significant strength of the current study, as most population-based studies on sleep rarely provide such detailed measures. Although selfreported sleep parameters, including SOL and WASO typically differ from those obtained from objective assessments, ${ }^{31}$ recent studies have shown that such self-report sleep assessments can be recommended for the characterisation of sleep parameters in both clinical and population-based research. ${ }^{32}$ Also, the accuracy of self-reported SOL and WASO are generally better among adolescents than in older adults, ${ }^{33}$ and a study of young adolescents in Hong Kong found good agreement between actigraphy measured and questionnaire reported sleep durations. ${ }^{34}$ The use of the Quantitative Research Criteria for Insomnia ${ }^{35}$ is also a major strength of the study, not limiting sleep problems to self-reported single items of initiating and maintaining sleep as has been used in previous studies. ${ }^{36}$ Self-harm was assessed without specifying the motivation(s) underpinning the behaviour. However, such operationalisation is consistent with clinical guidance ${ }^{37}$ and is employed widely in adolescent studies in Europe. ${ }^{15}$ Many previous studies have also focused on self-harm in relation to suicidal ideation or suicide attempts but the current study was restricted to self-harm. It should also be noted that our operationalisation of socioeconomic status included the adolescents' own perceived family economy, rather than objective measures of household income. However, this index of socioeconomic status has been shown to align well with previous studies in which family economy has been defined using more traditional methods. ${ }^{38}$ Also, the cross-sectional design restricts causal attributions, and longitudinal studies are needed to assess the temporal association between sleep, self-harm and depression.

Are the findings representative? We have previously demonstrated that the prevalence of insomnia and short sleep duration in the present study are at the higher end of the prevalence estimates in the literature. ${ }^{23}$ However, this can also be seen as a result of the shorter sleep duration in recent years. The prevalence estimates of self-harm were somewhat lower than in previous studies. This may partly be due to missing data, as some of the adolescents did not provide sufficiently specific information needed to code the self-harm acts. The inclusion of adolescents with incomplete information would yield prevalence rates more in line with previous studies. ${ }^{39}$ Also, depression was assessed by a self-report instrument, the SMFQ. As no validated cut-off exists for Norwegian adolescents, the 90th percentile on the total SFMQ score was chosen as an operationalisation of depression. Clearly, this does not imply the existence of a clinical diagnosis, such as major depressive disorder, and the lack of a clinical interview in confirming a clinical diagnosis of depression is a limitation of the present study. This is in contrast to conventional depression rating scales which normally contain such items, thereby preventing circularity and facilitating the unambiguous interpretation of associations between the symptoms of sleep and affective problems in the present study. Tiredness was included in the SMFQ; however, the association with several sleep parameters was not higher for this item than for other depressive symptoms.

Furthermore, although we did assess depression, perfectionism and ADHD, which accounted for some of the link between sleep and self-harm in the full model, there may be other covariates not addressed in the current study that may explain parts of this association, such as other mental health disorders (e.g. psychosis or bipolar disorder) or physical illnesses. Although beyond the scope of the present study, future research could usefully explore the relationship between cognitive variables (e.g. rumination and hopelessness), sleep problems and self-harm. Another limitation comprises the inclusion of a relatively low number of adolescents not in school compared with adolescents in school. It is worth noting, however, that many of the previous European studies of self-harm have excluded adolescents who are not in school. Finally, the attrition from the study could affect generalisability, with a response rate of about $53 \%$ and with adolescents in schools overrepresented. Based on previous research from the former waves of the Bergen Child Study, non-participants often have more psychological problems than participants, ${ }^{40}$ and it is therefore likely that the prevalence of both self-harm, sleep problems and depression may be underestimated in the current study.

Self-harm is related to sleep across a wide range of sleep parameters and this relationship is partly accounted for by depression. Addressing both sleep and depression in the prevention and treatment of self-harm may be fruitful avenues for new research.

Mari Hysing, PhD, PsyD, The Regional Centre for Child and Youth Mental Health and Child Welfare, Uni Research Health, Bergen, Norway; Børge Sivertsen, PhD, PsyD, The Regional Centre for Child and Youth Mental Health and Child Welfare, Uni Research Health, Bergen, Norway, Division of Mental Health, Norwegian Institute of Public Health, Bergen, and Department of Psychiatry, HelseFonna HF, Haugesund, Norway; Kjell Morten Stormark, PhD, PsyD, The Regional Centre for Child and Youth Mental Health and Child Welfare, Uni Research Health, Bergen, and Department of Clinical Psychology, University of Bergen, Norway; Rory C. O'Connor PhD, CPsychol, FAcSS, Suicidal Behaviour Research Laboratory, Institute of Health \& Wellbeing, University of Glasgow, UK

Correspondence: Mari Hysing, Regional Centre for Child and Youth Mental Health and Child Welfare, Postboks 7810, 5020 Bergen, Norway. Email: mari.hysing@uni.no

First received 14 Feb 2014, final revision 25 Nov 2014, accepted 27 Nov 2014 


\section{References}

1 Hawton K, Saunders KE, O'Connor RC. Self-harm and suicide in adolescents. Lancet 2012; 379: 2373-82.

2 O'Connor RC, Rasmussen S, Hawton K. Distinguishing adolescents who think about self-harm from those who engage in self-harm. Br J Psychiatry 2012 200: $330-5$.

3 de Wilde E. Adolescent suicidal behaviour: a general population perspective. In The International Handbook of Suicide and Attempted Suicide (eds K Hawton, K van Heeringen): 249-60. John Wiley \& Sons, 2000.

4 Klonsky E, Glenn C. Assessing the functions of non-suicidal self-injury: psychometric properties of the Inventory of Statements About Self-injury (ISAS). J Psychopathology Behav Assess 2009; 31: 215-9.

5 Wong MM, Brower KJ, Zucker RA. Sleep problems, suicidal ideation, and self-harm behaviors in adolescence. J Psychiatr Res 2011; 45: 505-11.

6 Carskadon MA, Acebo C, Jenni OG. Regulation of adolescent sleep: implications for behavior. Ann N Y Acad Sci 2004; 1021: 276-91.

7 Dahl RE, Lewin DS. Pathways to adolescent health sleep regulation and behavior. J Adolescent Health Off Publ Soc Adolescent Med 2002; 31: 175-84.

8 Wong MM, Brower KJ. The prospective relationship between sleep problems and suicidal behavior in the National Longitudinal Study of Adolescent Health. J Psychiatr Res 2012; 46: 953-9.

9 Singareddy R, Krishnamurthy VB, Vgontzas AN, Fernandez-Mendoza J, Calhoun SL, Shaffer ML, et al. Subjective and objective sleep and self-harm behaviors in young children: a general population study. Psychiatry Res 2013; 209: 549-53.

10 Lundh LG, Bjarehed J, Wangby-Lundh M. Poor sleep as a risk factor for nonsuicidal self-injury in adolescent girls. J Psychopathology Behav Assess 2013; 35: 85-92.

11 Fujino $\mathrm{Y}$, Mizoue T, Tokui N, Yoshimura T. Prospective cohort study of stress, life satisfaction, self-rated health, insomnia, and suicide death in Japan. Suicide Life Threat Behav 2005; 35: 227-37.

12 Fawcett J, Scheftner WA, Fogg L, Clark DC, Young MA, Hedeker D, et al. Time-related predictors of suicide in major affective disorder. Am J Psychiatry 1990; 147: 1189-94.

13 Allely CS. The association of ADHD symptoms to self-harm behaviours: a systematic PRISMA review. BMC Psychiatry 2014; 14: 133.

14 O'Connor RC. The relations between perfectionism and suicidality: a systematic review. Suicide Life-Threat 2007; 37: 698-714.

15 Madge N, Hewitt A, Hawton K, de Wilde EJ, Corcoran P, Fekete S, et al. Deliberate self-harm within an international community sample of young people: comparative findings from the Child \& Adolescent Self-harm in Europe (CASE) Study. J Child Psychol Psychiatry 2008; 49: 667-77.

16 American Psychiatric Association. Diagnostic and Statistical Manual of Mental Disorders (Fifth ed.). 4th ed. American Psychiatric Publishing, 2013.

17 Thapar A, McGuffin P. Validity of the shortened Mood and Feelings Questionnaire in a community sample of children and adolescents: a preliminary research note. Psychiatry Res 1998; 81: 259-68.

18 Sharp C, Goodyer IM, Croudace TJ. The Short Mood and Feelings Questionnaire (SMFQ): a unidimensional item response theory and categorical data factor analysis of self-report ratings from a community sample of 7-through 11-year-old children. J Abnormal Child Psychol 2006; 34: 379-91.

19 Lundervold AJ, Posserud M, Stormark K, Breivik B, Hysing M. Symptoms of depression as reported by Norwegian adolescents on the Short Mood and Feelings Questionnaire. Front Psychol 2013; 4: 613.

20 Garner DM, Olmsted MP, Polivy J. Eating disorder inventory. Psychopharmacol Bull 1985; 21: 1009-10.
21 Kessler RC, Adler LA, Gruber MJ, Sarawate CA, Spencer T, Van Brunt DL. Validity of the World Health Organization Adult ADHD Self-Report Scale (ASRS) Screener in a representative sample of health plan members. Int J Methods Psychiatr Res 2007; 16: 52-65.

22 Adler LA, Shaw DM, Spencer TJ, Newcorn JH, Hammerness P, Sitt DJ, et al. Preliminary examination of the reliability and concurrent validity of the attention-deficit/hyperactivity disorder self-report scale v1.1 symptom checklist to rate symptoms of attention-deficit/hyperactivity disorder in adolescents. J Child Adolesc Psychopharmacol 2012; 22: 238-44.

23 Hysing M, Pallesen S, Stormark KM, Lundervold AJ, Sivertsen B. Sleep patterns and insomnia among adolescents: a population-based study. J Sleep Res 2013; 22: 549-56.

24 Azevedo MH, Bos SC, Soares MJ, Marques M, Pereira AT, Maia B, et al. Longitudinal study on perfectionism and sleep disturbance. World J Biol Psychiatry Off J World Fed Soc Biol Psychiatry 2010; 11: 476-85.

25 Cassoff J, Wiebe ST, Gruber R. Sleep patterns and the risk for ADHD: a review. Nature Sci Sleep 2012; 4: 73-80.

26 Sivertsen B, Harvey AG, Lundervold AJ, Hysing M. Sleep problems and depression in adolescence: results from a large population-based study of Norwegian adolescents aged 16-18 years. Eur Child Adolesc Psychiatry 2013; 23: 681-9.

27 Talbot LS, McGlinchey EL, Kaplan KA, Dahl RE, Harvey AG. Sleep deprivation in adolescents and adults: changes in affect. Emotion 2010; 10: 831-41.

28 O'Connor RC, Rasmussen S, Miles J, Hawton K. Self-harm in adolescents: self-report survey in schools in Scotland. Br J Psychiatry 2009; 194: 68-72.

29 Harvey AG. A transdiagnostic approach to treating sleep disturbance in psychiatric disorders. Cogn Behav Ther 2009; 38 (suppl 1): 35-42.

30 Brent DA, McMakin DL, Kennard BD, Goldstein TR, Mayes TL, Douaihy AB. Protecting adolescents from self-harm: a critical review of intervention studies. J Am Acad Child Adolesc Psychiatry 2013; 52: 1260-71.

31 Lauderdale DS, Knutson KL, Yan LL, Liu K, Rathouz PJ. Self-reported and measured sleep duration: how similar are they? Epidemiology 2008; 19: 838-45.

32 Zinkhan M, Berger K, Hense S, Nagel M, Obst A, Koch B, et al. Agreement of different methods for assessing sleep characteristics: a comparison of two actigraphs, wrist and hip placement, and self-report with polysomnography. Sleep Med 2014; 15: 1107-14.

33 Dillon HR, Lichstein KL, Dautovich ND, Taylor DJ, Riedel BW, Bush AJ. Variability in self-reported normal sleep across the adult age span. $J$ Gerontol B Psychol Sci Soc Sci 2015; 70: 46-56.

34 Kong AP, Wing YK, Choi KC, Li AM, Ko GT, Ma RC, et al. Associations of sleep duration with obesity and serum lipid profile in children and adolescents. Sleep Med 2011; 12: 659-65.

35 Lichstein KL, Durrence HH, Taylor DJ, Bush AJ, Riedel BW. Quantitative criteria for insomnia. Behav Res Ther 2003; 41: 427-45.

36 Meyer KA, Wall MM, Larson NI, Laska MN, Neumark-Sztainer D. Sleep duration and BMI in a sample of young adults. Obesity (Silver Spring) 2012; 20: $1279-87$.

37 Kapur N, Cooper J, O'Connor RC, Hawton K. Non-suicidal self-injury v. attempted suicide: new diagnosis or false dichotomy? Br J Psychiatry 2013; 202: $326-8$

38 Bøe T, Sivertsen B, Heiervang E, Goodman R, Lundervold AJ, Hysing M Socioeconomic status and child mental health: the role of parental emotional well-being and parenting practices. J Abnormal Child Psychol 2014; 42: 705-15.

39 Muehlenkamp JJ, Claes L, Havertape L, Plener PL. International prevalence of adolescent non-suicidal self-injury and deliberate self-harm. Child Adolesc Psychiatry Ment Health 2012; 6: 10.

40 Stormark KM, Heiervang E, Heimann M, Lundervold A, Gillberg C. Predicting nonresponse bias from teacher ratings of mental health problems in primary school children. J Abnorm Child Psychol 2008; 36: 411-9. 